\title{
Fractionated Mapping-Guided Ablation Strategy in Patients with Long-Standing Persistent Atrial Fibrillation: a Case Series
}

Tolga Aksu', ${ }^{1}$, Tumer Erdem Guler ${ }^{1}$, Serdar Bozyel ${ }^{1}$, Kivanc Yalin²

\section{ORCID IDs}

Aksu T (1) https://orcid.org/0000-0001-8061-9660

Guler TE (1) https://orcid.org/0000-0003-1349-2123

Bozyel S (1) https://orcid.org/0000-0002-6582-3660

Yalin K (10) https://orcid.org/0000-0001-5602-0711

\begin{abstract}
Although pulmonary vein isolation (PVI) remains the cornerstone of ablation for paroxysmal atrial fibrillation (AF), optimal ablation strategy for long-standing persistent AF (LSPAF) remains unclear. This article presents two patients with LSPAF in whom acute AF termination was achieved during ablation by using fractionated-guided extended PVI, posterior wall isolation, and mitral isthmus
\end{abstract}

KEYWORDS: Atrial fibrillation; Cardiac ablation procedures; Arrhythmia.

1.University of Health Sciences - Kocaeli Derince Training and Research Hospital - Department of Cardiology - Kocaeli - Turkey.

2.Istanbul University-Cerrahpasa - Faculty of Medicine - Department of Cardiology - Istanbul - Turkey.

*Correspondence author: aksutolga@gmail.com

Received: 08 April 2020 | Accepted: 08 April 2020 


\section{INTRODUCTION}

Although pulmonary vein isolation (PVI) is considered as the cornerstone of ablation for paroxysmal atrial fibrillation (AF), there is still no well-accepted ablation strategy for long-standing persistent AF (LSPAF) ${ }^{1}$. To detect possible sources for perpetuation of AF fractionated electrograms have been used by different groups for a long time ${ }^{2}$. However, ablation at sites demonstrating fragmentation did not always result in prolongation of AF cycle length or arrhythmia termination, which indicates that some fractionated electrograms may be active drivers, while others are likely passive effect of fibrillatory conduction. As a newly investigated tool, the potential usage of fractionation mapping of the software EnSite Precision (Abbott) mapping system was firstly tested to detect critical substrate during $\mathrm{AF}$ by our group 3 .

Herein, this report presented two patients with LSPAF in whom acute AF termination was achieved by stepwise ablation covering electrograms exhibiting fractionation pattern.

\section{CASE 1}

A 48-year-old woman with a history of hypertrophic cardiomyopathy (left ventricular ejection fraction of $65 \%$, left atrial anteroposterior diameter of $5.1 \mathrm{~cm}$ ) was admitted to our outpatient clinic due to drug resistant symptomatic LSPAF. Sinus rhythm could not be maintained despite numerous electrical or pharmacological cardioversion attempts.

She received a dual-chamber implantable cardioverter-defibrillator for primary prevention of sudden cardiac death in 2014. She was deemed an appropriate candidate for catheter ablation with the intention to minimize high ventricular rate related symptoms.

\section{Details of mapping}

Following transseptal puncture, fractionation mapping of left atrium was performed by using an Inquiry AFocusII (Abbott) multielectrode catheter with $4 \mathrm{~mm}$ electrode spacing.

Fractionation mapping tool is a software to detect fragmentation pattern based on the number of fractionations per unit of time, sites exhibiting discrete atrial complexes and consistent activation sequence. Unlike the classical complex fractionated atrial electrograms (CFAE), mean mapping tool of EnSite system, cycle length of AF is overlooked in this method (Fig. 1). Map parameters were standardized at internal and external projections of $7 \mathrm{~mm}$ and interpolation of $7 \mathrm{~mm}$, low-voltage identification of $0.1 \mathrm{mV}$. To exclude background system noise, low threshold of $0.05 \mathrm{mV}$ was applied. Voltage peaks greater than this threshold but less than $0.1 \mathrm{mV}$ were defined as scar tissue. Fractionation map was created using following parameters of width $(5 \mathrm{~ms})$, refractory time (30 s), roving sensitivity $(0.1 \mathrm{mV}$ ), and fractionation threshold (4 and 6) (Fig. 2). Based on width, refractory period, and sensitivity, a fractionation score was assigned to each electrogram by the mapping algorithm. The areas with a fractionation score above 4, which were suggestive of a fractionated electrogram, were accepted as potential drivers for AF. The map color scale was set to color areas at or above the defined fractionation threshold as purple (suggestive of fractionated electrogram), and below as white (suggestive of nonfractionated electrogram).

Before performing stepwise ablation consisting of circumferential PVI, roof and floor lines for posterior wall isolation, and mitral isthmus lines, ablation lines were predetermined to cover fragmented areas (Fig. 3). 


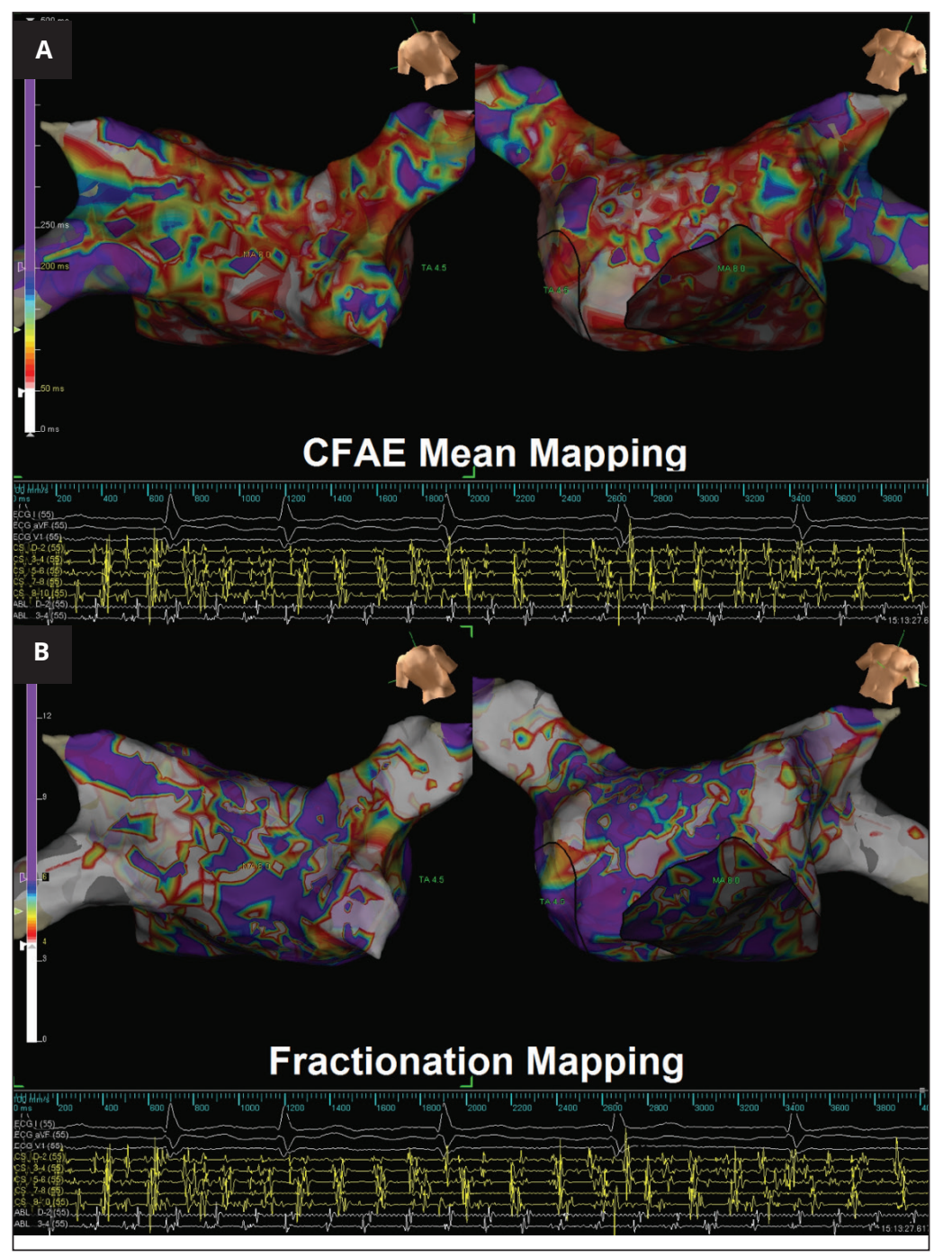

Figure 1. Difference of complex fractionated atrial electrograms (CFAE) mean and fractionation mapping tools of EnSite system. Note: Although fractionation is determined by the average interval between deflections and is considered to occur when the mean is $\leq 120 \mathrm{~ms}$ over $5 \mathrm{~s}$ in the CFAE mean module, fractioned mapping tool does not take into account the cycle length of AF and only shows fragmented areas.

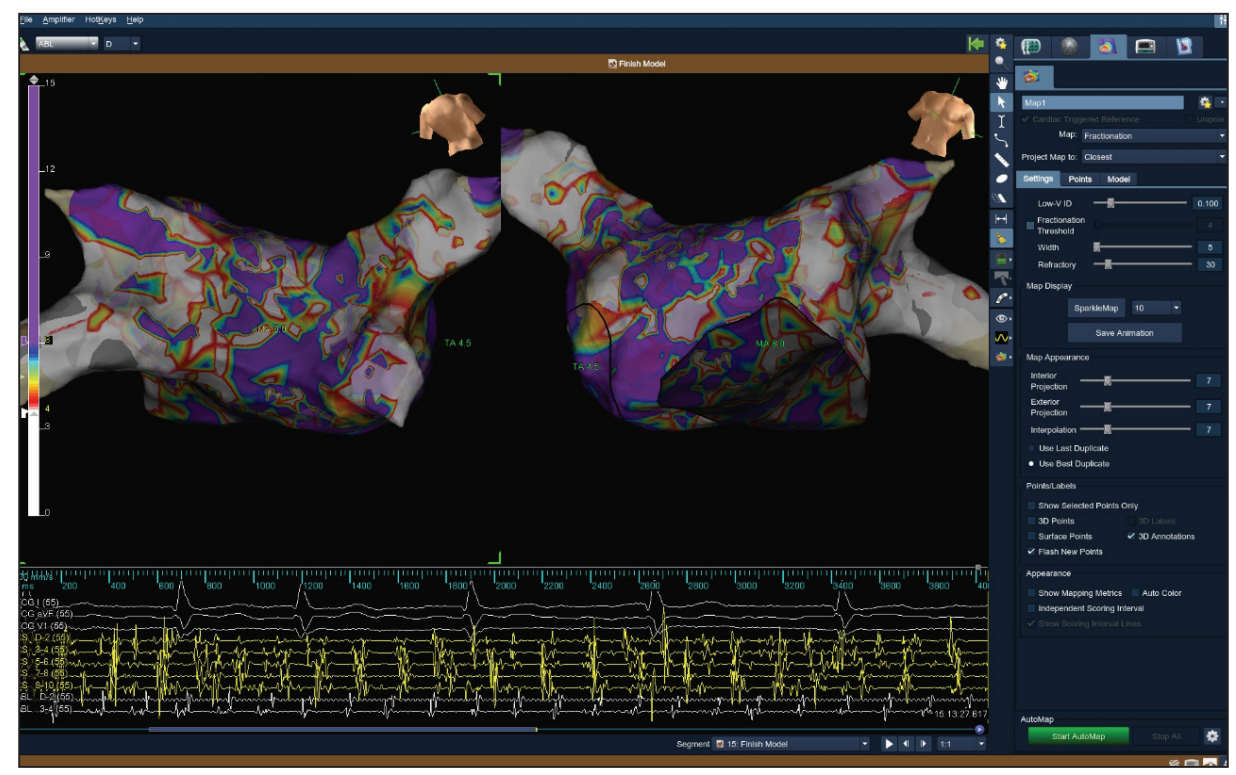

Figure 2. Preparation of fractionation map parameters for data collection in Case 1. 


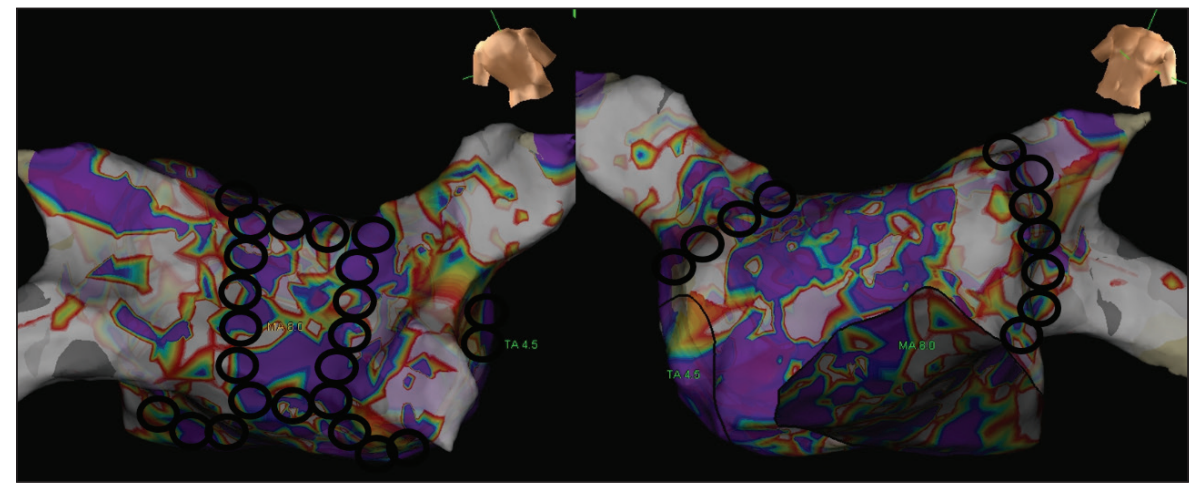

Figure 3. Predefinition of ablation lines consisting of pulmonary vein isolation and box isolation for posterior wall according to fragmented areas in Case 1.

\section{Details of ablation}

Radiofrequency energy was applied with a target temperature of less than $42{ }^{\circ} \mathrm{C}$ and target power of $30-40 \mathrm{~W}$ until obtaining $>80 \%$ reduction of electrical potentials amplitude and at least $10 \mathrm{ohms}$ in impedance drop. During the circumferential PVI procedure, ablation lines were extended toward purple areas on the fractionation map as much as possible. During the circumferential PVI procedure for the left pulmonary veins, a significant cycle length prolongation, conversion to atrial macroreentry and occurrence of sinus rhythm were seen, respectively (Video 1).

Following completion of the circumferential PVI procedure, electrical isolation and bidirectional block was confirmed in all the pulmonary veins. Atrial fibrillation tried to be induced by rapid atrial pacing from distal tip of the coronary sinus catheter. Pacing was applied at the shortest 1:1 atrial capture rate for up to $10 \mathrm{~s}$ at a time. No AF was inducible with and without isoproterenol infusion.

Because there was no significant scar tissue in posterior wall, further ablation was not applied for isolation of posterior wall. No complication was encountered. At 3, 6, and 12 months after the procedure, outpatient visits involving 12-lead electrocardiography, 72-hour Holter monitoring, and transthoracic echocardiography was conducted. At 12-month follow-up, the patient remains symptom free and in normal sinus rhythm on a low dose of metoprolol (50 $\mathrm{mg}$ twice a day) and warfarin for stroke prevention. Left atrial diameter were detected as $4.6 \mathrm{~cm}$ on follow-up echocardiography.

\section{CASE 2}

A 72-year-old man presented with a history of AF for more than 12 years. Transthoracic echocardiogram noted mild left ventricular systolic dysfunction with left ventricular ejection fraction of $45 \%$ and mild-to-moderate mitral regurgitation. He has had a couple of admissions for decompensated heart failure in the setting of AF with rapid ventricular response despite usage of optimal beta-blocker and amiodarone. However, concern remained about long-term amiodarone use, and the decision was made to undergo catheter ablation.

\section{Details of mapping and ablation}

Fractionation mapping during AF was done as described in case 1 (Figs. 4 and 5). Following completion of the circumferential PVI procedure, the patient was still in AF. Therefore, roof and floor lines were applied to achieve posterior wall isolation. During the posterior wall isolation, ablation lines were extended toward purple areas on the fractionation map as much as possible. A significant $\mathrm{AF}$ cycle length prolongation and occurred during extended ablation on the floor line (Video 2). The coronary sinus recordings demonstrated a regularization of $\mathrm{AF}$ to atrial flutter during completion of floor and roof lines. The activation mapping of right atrium was recorded using the circular mapping catheter. The intracardiac signals and activation mapping revealed clockwise typical atrial flutter and positive concealed entrainment verified that the tachycardia was isthmus dependent (Video 3). Ablation of cavotricuspid isthmus 
was performed using a point-by-point approach for $60 \mathrm{~s}$ at each point with a power limit of $45 \mathrm{~W}$ and a target temperature of $42^{\circ} \mathrm{C}$. The patient turned to normal sinus rhythm during ablation. The presence of bidirectional block was confirmed with the appropriate maneuvers. No AF or atrial tachycardia was inducible with coronary sinus stimulation with and without isoproterenol infusion.

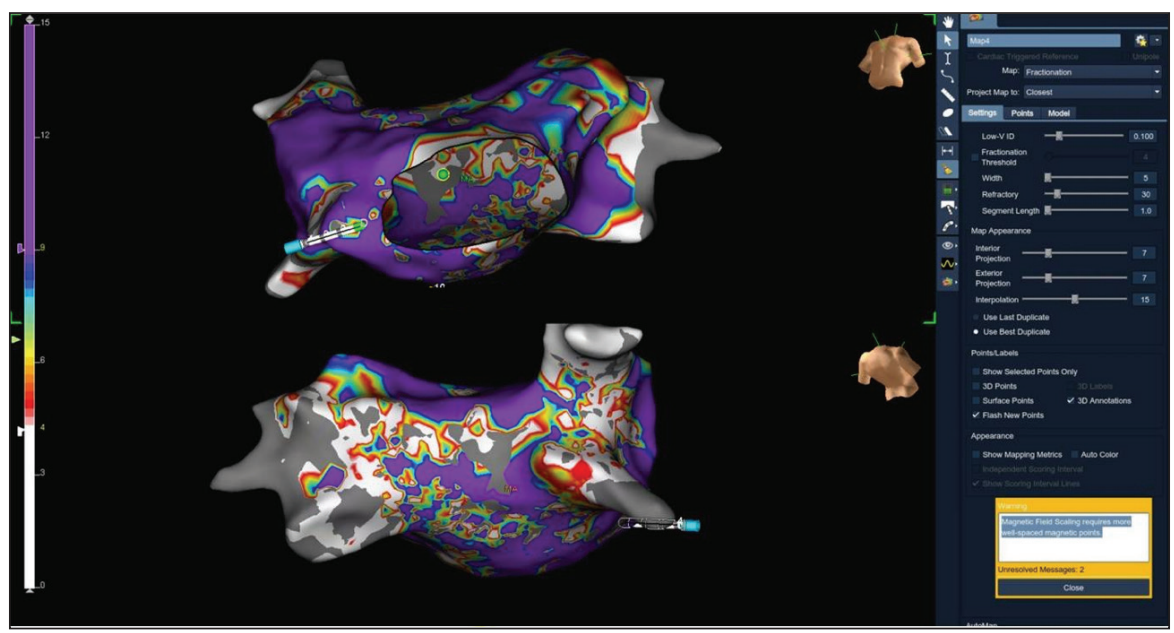

Figure 4. Preparation of fractionation map parameters for data collection in Case 2.

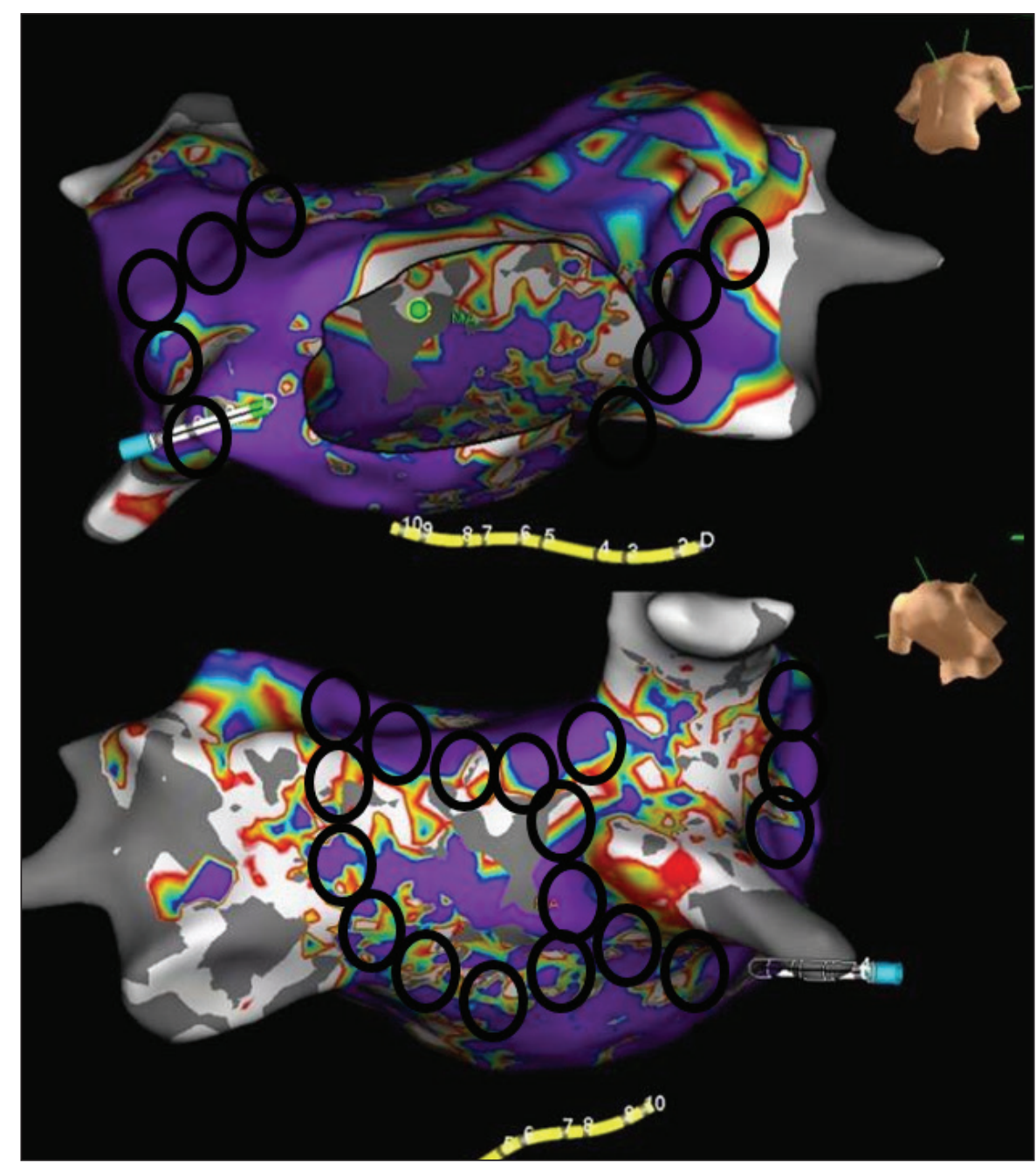

Figure 5. Predefinition of ablation lines consisting of pulmonary vein isolation and box isolation for posterior wall according to fragmented areas in Case 2. 


\section{DISCUSSION}

In a pilot study presented as oral abstract, the authors of this report investigated possible role of extended PVI, posterior wall isolation, and mitral isthmus lines to cover electrograms exhibiting fractionation pattern during stepwise ablation in acute AF termination rate and in long term success in patients with LSPAF5. Twelve patients with LSPAF underwent fractionation mapping-guided stepwise ablation. Acute AF termination was seen in 8 of 12 patients. Sinus was achieved by electrical cardioversion in remaining 3 cases. At a mean 1-year follow-up, all the patients demonstrating acute termination remained in sinus rhythm without antiarrhythmic drugs. Atrial tachycardia was seen in 2 of 3 cases in whom sinus was achieved by cardioversion. Details of the current ablation strategy in patients with persistent $\mathrm{AF}$ and LSPAF are provided in Fig. 6.

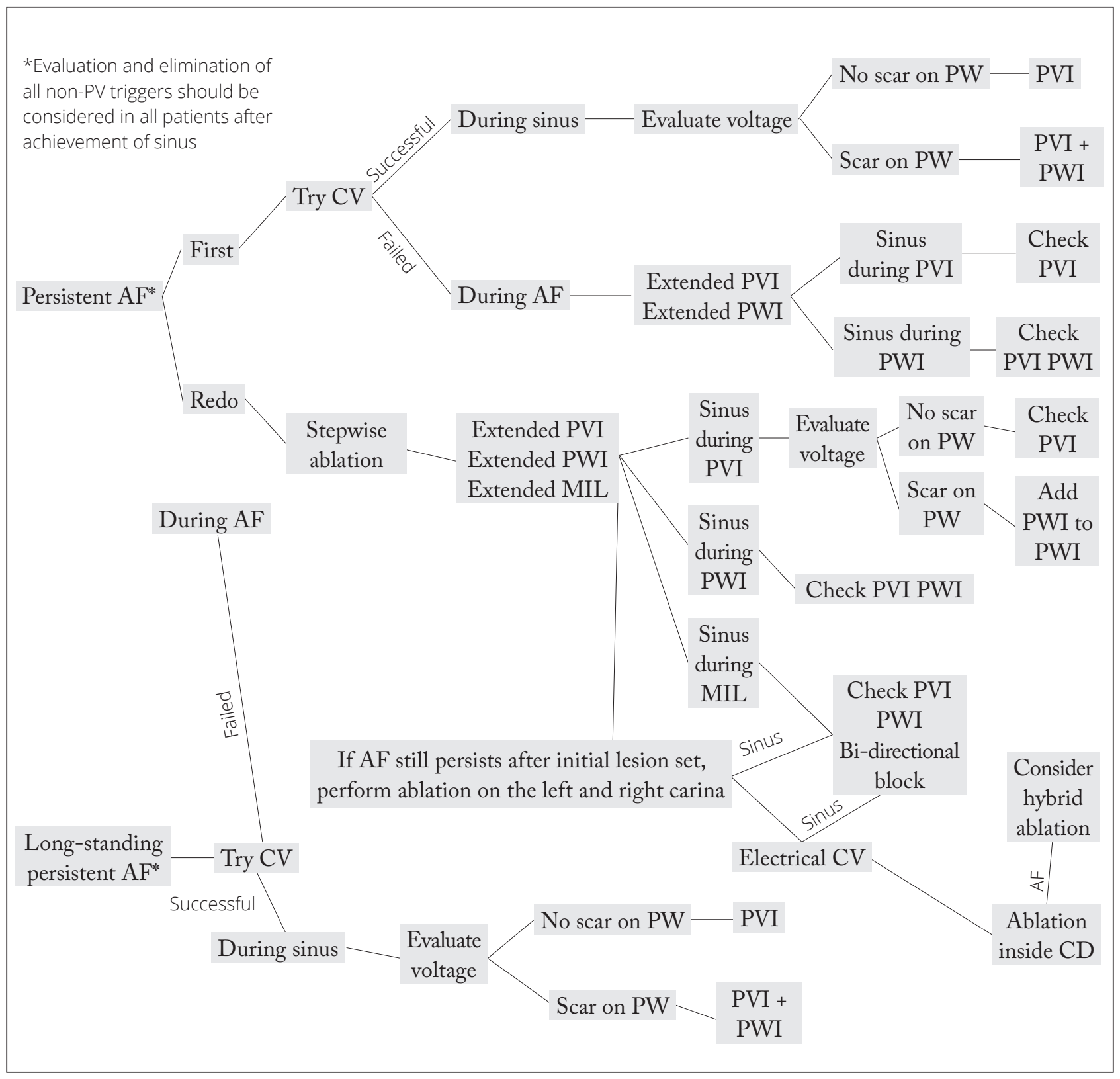

Figure 6. Ablation strategy in patients with persistent and long-persistent atrial fibrillation. 


\section{AUTHOR'S CONTRIBUTION}

Conceptualization, Aksu T. and Guler T. E.; Methodology, Aksu T.; Investigation, Aksu T. and Bozyel S.; Writing Original Draft, Aksu T. and Yalin K.; Writing - Review and Editing, Aksu T.

\section{REFERENCES}

1. Calkins H, Hindricks G, Cappato R, Kim Y-H, Saad EB, Aguinaga L, et al. 2017 HRS/EHRA/ECAS/APHRS/SOLAECE expert consensus statement on catheter and surgical ablation of atrial fibrillation. Heart Rhythm. 2017;14(10):e275-444. https://doi.org/10.1016/j. hrthm.2017.05.012

2. Aksu T, Guler TE, Yalin K, Oto A. Unanswered questions in complex fractionated atrial electrogram ablation. Pacing Clin Electrophysiol. 2016;39(11):1269-78. https://doi.org/10.1111/pace.12944

3. Aksu T, Guler TE, Bozyel S, Yalin K. Usage of a new mapping algorithm to detect possible critical substrate for continuity of atrial fibrillation: fractionation mapping in: preliminary experience. J Interv Card Electrophysiol. 2020;58(1):29-34. https://doi.org/10.1007/ s10840-019-00693-x

4. Aksu T, Guler TE. Electroanatomical mapping-guided ablation during atrial fibrillation: a novel usage of fractionation mapping in a case with sinus bradycardia and paroxysmal atrial fibrillation. J Interv Card Electrophysiol. 2020;57(2):331-2. https://doi.org/10.1007/ s10840-019-00633-9

5. Aksu T, Guler TE, Bozyel S. This Supplement contains the selected Abstracts presented at the Venice Arrhythmias 2019, held October 3-5, 2019. Clinical outcome of fractionation mapping guided ablation after failed electrical cardioversion in patients with long-standing persistent atrial fibrillation: a pilot study. J Interv Card Electrophysiol. 2020;57:163-205. https://doi.org/10.1007/ s10840-019-00665-1 